\title{
Adaptive Transcoders for Video \& Image Sequence Using Wavelet Transform
}

\author{
T. Arumuga Maria Devi ${ }^{1}$, K.K Sherin ${ }^{2}$ \\ ${ }^{1}$ Assistant Professor, ${ }^{2}$ Assistant Professor \\ ${ }^{1}$ Centre for Information Technology and Engineering, Manonmaniam Sundaranar University, Tirunelveli \\ ${ }^{2}$ Bethlahem Institute of Engineering, Affiliated to Anna University, Chennai
}

\begin{abstract}
This paper deals mainly with the performance study and analysis of sending and retrieving an image across Client/Server network. The image that is to be send across the network should be compressed in order to transfer it at a minimal amount of time. Other than that, the image when compressed will suffer loss in the image quality. The main work identified are that efficient retrieval of an image will be made possible using Wavelet transform and various other methods such as Transcoding etc. These methods used above to download an image from a distant location are found to be more efficient in comparison with the other image downloading techniques.
\end{abstract}

Keywords - 5/3 Wavelet Transform, Significant Mapping, Mosaicking, Refinement Mapping, Transcoding.

\section{INTRODUCTION}

The transfer of images in wireless networks presents major challenges which raises issues related to its representation, its storage and its transmission. The challenges of image transmission includes limited bandwidth of cellular networks, the restriction in computational power, limited storage capability, and constraints related to the power sources of the appliances. To handle the problem, various methods are used. The preprocessing using wavelet based compression methodology is used. An efficient Wavelet based compression can significantly minimize the energy required for wireless image communication while meeting bandwidth constraints of wireless and network image quality. Based on Discrete Wavelet Transform, an efficient image compression scheme is used by enabling significant reduction in computation energy needed with minimal degradation of image quality. Wavelet transforms are based on small waves, called wavelets, of varying frequency and limited duration. The 5/3 wavelet transform is well suited for mosaic images, the interleaving of color components are automatically taken care and it transforms as a smooth channel without de-interleaving. The 5/3 wavelet transforms requires the least computation among the various reversible integer to integer wavelet transformations. Transcoding means a process for choosing, changing, or producing the content (text, image, voice and video) to be conformed with the current environment that user prefers. This can be achieved by changing the size of an image, its resolution and quality, replacing voice by its text or video by a number of images. Transcoding makes web pages more userfriendly and show them appropriately in different mobile devices with different screen sizes, reducing size of web pages in order to reduce network bandwidth and decreasing their loading time. Current Transcoding systems achieve these goals by either removing non-vital parts of web pages such as pictures or content to the required format on the mobile advertisements, or moving some content from one page to device.

\section{TECHNIQUE}

The problem lay in the past decades in sending the images through a Client/Server network in a minimal amount of time. If the image is having a larger amount of size, the problem was that the image required compression. If compression is used, some amount of information in the image will be lost thus leading to the reduction in the quality of an image. So, the primary importance was that to transmit the image within a minimal amount of time. Various methods such as wavelet transform are used for compressing the image. With Wavelet transform based compression, the quality of compressed images is typically high, and the option of a perfect compression ratio is complicated to formulate as it varies depending on the content of the image. The fundamental approach to image compression consists of a number of key steps. They are wavelet packet decomposition, quantization, and organization of vectors, lossless encoding and reduction. As an initial stage of image compression, the image is put through several layers of wavelet packet decomposition. The results of the decomposition are then divided or processed in some way, depending on the method. Integer quantization is performed on all of the decomposed wavelet sections. The quantization value is the determining factor of quality. A quantization value of 1 is near lossless quality, although little to no compression is achieved. This is accomplished by taking each section and dividing it by a set value and rounding to the nearest integer. Images are decomposed using wavelet filters into a set of sub bands with different resolution corresponding to different frequency bands. Different quantization and coding schemes are used for different sub bands based on their statistical properties. Wavelet transform make use of both the spatial and frequency correlation of data by dilations (or contractions) and translations of mother wavelet on the input data. It supports the multi resolution analysis of data i.e. it can be applied to various scales according to the details required, which allows progressive transmission and zooming of the image without the need of extra storage. One more encouraging feature of wavelet transform is its symmetric behavior that is both the forward and the inverse transform has the same complexity, building fast compression and decompression routines. Its characteristics well suited for image compression comprise the ability to take into account of Human Visual System's (HVS) characteristics, very good energy compaction capabilities, robustness under transmission, high compression ratio etc. The implementation of wavelet compression method is very similar to that of sub band coding scheme: the signal is decomposed using filter banks. The output of the filter banks is down-sampled, quantized, and encoded. The decoder decodes the coded representation, up-samples and recomposes the 
signal. Wavelet transform divides the information of an image into approximation and detail sub signals. The approximation sub signal illustrates the general trend of pixel values and other three detail sub signals show the vertical, horizontal and diagonal details or changes in the images. If these details are very small (threshold) then they can be set to zero without significantly changing the image. The greater the number of zeros the greater the compression ratio. If the energy retained (amount of information retained by an image after compression and decompression) is 100\% then the compression is lossless as the image can be reconstructed exactly. This occurs when the threshold value is set to zero, meaning that the details have not been changed. If any value is changed then energy will be lost and thus lossy compression occurs. As more zeros are obtained, more energy is lost. Therefore, a balance between the two is needed.

\section{Image Mosaicking}

Image Mosaicking is the process of reconstructing or stitching a single, continuous image from a set of separate or overlapped sub-images. The various methods adopted for image mosaicking can be broadly classified into;

1.) Direct methods

2.) Feature based methods.

Direct methods are found to be useful for mosaicking large overlapping regions, small translations and rotations. Feature based methods can usually handle small overlapping regions and in general tend to be more accurate but computationally intensive. Some of the basic problems in image mosaicking are Global alignment, Local adjustment, Automatic selection, Image blending, Auto exposure compensation.

Since the image may have incomplete color samples, reconstructing the image is necessary. For this purpose, DeMosaicking algorithm is used. It should be able to avoid false color artifacts such as chromatic aliases, should preserve image resolution, should have low computational complexity and also should have an availability to reduce the noise.

The algorithms that are implemented in De-mosaicking are Nearest neighbor color and Wavelet Based Demosaicking algorithms.

\section{Discrete Wavelet Transform}

Discrete Wavelet Transform (DWT) is the most popular transform for image-based application [16], [18], [20]. A 2-dimensional wavelet transform is applied to the original image in order to decompose it into a series of filtered sub band images. At the top left of the image is a low-pass filtered version of the original and moving to the bottom right, each component contains progressively higher-frequency information that adds the detail of the image. It is clear that the higherfrequency components are relatively sparse, i.e., many of the coefficients in these components are zero or insignificant. The wavelet transform is thus an efficient way of decorrelating or concentrating the important information into a few significant coefficients. The wavelet transform is particularly effective for still image compression and has been adopted as part of the JPEG 2000 standard [6] and for still image texture coding in the MPEG-4 standard.

The Discrete Wavelet Transform of a signal, $x(t)$ is defined to be the set of analysis coefficients:

Analysis: $\mathrm{c}_{\mathrm{j}, \mathrm{k}}=\int_{-\infty}^{\infty} \mathrm{x}(\mathrm{t}) \mathrm{w}_{\mathrm{j}, \mathrm{k}}(\mathrm{t}) \mathrm{dt}$

From these, the signal is recovered as,

Synthesis: $\mathrm{x}(\mathrm{t})=\sum_{\mathrm{j}} \sum_{\mathrm{k}} \mathrm{c}_{\mathrm{j}, \mathrm{k}} \mathrm{w}_{\mathrm{j}, \mathrm{k}}(\mathrm{t})$

Assuming the existence of a scaling function $\Phi(\mathrm{t})$, we can now modify this definition as follows. Since the spaces $w_{j}$ are getting larger and larger as $j$ goes to $\infty$, we can approximate any signal, $x(t)$, closely by choosing a large enough value of $\mathrm{j}=\mathrm{J}$ and projecting the signal into $w_{j}$ using the basis, $\Phi_{\mathrm{j}, \mathrm{m}}(\mathrm{t})$ (all values of $k$ ).

$\mathrm{cA}_{0} \mathrm{~m}: \int_{-\infty}^{\infty} \mathrm{x}(\mathrm{t}) \Phi_{\mathrm{j}, \mathrm{m}}(\mathrm{t}) \mathrm{dt}$

From these we can approximately recover the signal as:

$\mathrm{x}(\mathrm{t}) \approx \sum_{\mathrm{m}} \mathrm{cA}_{0} \mathrm{~m} \Phi_{\mathrm{j}, \mathrm{m}}(\mathrm{t})$

In effect, we replace the signal, $x(t)$, by the approximate signal given by the projection coefficients, $\mathrm{cA}_{0} \mathrm{~m}$. After this approximation our signal is now in $v_{j}$ and we can decompose it using the subspaces $v_{j-n}$ and $w_{j-n}$ with their bases $\Phi_{j-n}, k(t)$ and $\mathrm{w}_{\mathrm{j}-\mathrm{n}, \mathrm{k}}(\mathrm{t})$. Note that the scale is getting larger and larger as the index $j-n$ gets more negative. If we take $n=1$, we get:

$\mathrm{v}_{\mathrm{j}}=\mathrm{w}_{\mathrm{j}-1}+\mathrm{v}_{\mathrm{j}-1}$

Using the basis $\mathrm{w}_{\mathrm{j}-1, \mathrm{k}}(\mathrm{t})$ in $\mathrm{w}_{\mathrm{j}-1}$ and $\Phi_{\mathrm{j}, 1, \mathrm{k}}(\mathrm{t})$ in $\mathrm{v}_{\mathrm{j}-1}$ we have:

$$
\begin{aligned}
\mathrm{x}(\mathrm{t})= & \sum_{\mathrm{m}} \mathrm{cA}_{0}(\mathrm{~m}) \Phi_{\mathrm{j}, \mathrm{m}}(\mathrm{t}) \\
& \sum_{\mathrm{k}} \mathrm{cA}_{1}(\mathrm{k}) \Phi_{\mathrm{j}-1, \mathrm{k}}(\mathrm{t})+\sum_{\mathrm{k}} \mathrm{cD}_{1}(\mathrm{k}) \mathrm{w}_{\mathrm{j}-1, \mathrm{k}}(\mathrm{t}) \\
& \mathrm{A}_{1}(\mathrm{t})+\mathrm{D}_{1}(\mathrm{t})
\end{aligned}
$$

As before, we call the signals $A_{1}(t)$ and $D_{1}(t)$ the approximation and detail and we call the coefficients $C_{1}(k)$ and $C_{1}(k)$ the approximation coefficients and the detail coefficients at level 1 . We can further decompose $\mathrm{A}_{1}(\mathrm{t})$ to get: 


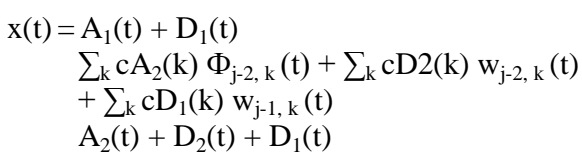

We call the signals $A_{2}(t)$ and $D_{2}(t)$ the approximation and details and we call the coefficients $c A_{2}(k)$ and $c D_{2}(k)$ the approximation coefficients and the detail coefficients at level 2 . We can continue in this way to decompose our signal further and further such as given below:

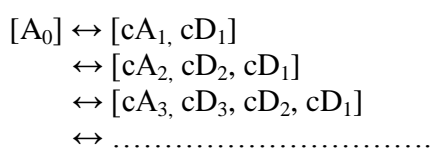

\section{Preprocessing}

In order to improve the quality of the image, one or more filtered versions of the image are stored in addition to the original patterns. This method is known as pre-processing. Pre-processing the images along with wavelet transform improves the quality of the image. As per the requirement, the image is broken down into sub images. This is the procedure done before processing which is to correct the image from different errors. This has to be done before image enhancement. Edges detection and noise reduction are a crucial and very important pre-processing step. The classical edge detection methods and filtering are less accurate in detecting complex edge and filtering various types of noise. Pre-processing is very useful in a variety of situations since it helps to suppress information that is not related to the exact image processing or analysis task. Image filtering and edge detection is an indispensable and necessary operation in image pre-processing step. When the image is acquired, the factors such as the projection, mix and noise are produced. These factors can lead to image blurring and distortion; consequently it can cause difficulty in extracting important feature in the image like edges of objects in image. Mathematical morphology is a mathematical method using structural elements to analyze image, which has been widely applied to variety fields like visual inspection. The morphological operations work to simplify image and preserve the main shape characteristics of objects. Morphological operators are mainly used in image preprocessing for noise filter, shape simplification and enhancing object structure. Mathematical morphology is a powerful tool to filter different types of noise and to detect the complex edge in variety directions.

\section{Refinement Mapping}

Lossless compression schemes with progressive transmission capability play a key role in critical data storage, retrieval and transmission, when a large volume of data must be handled fast, without distortions, over large networks with limited bandwidth. The standard wavelet compression techniques, even if loss-less in principle, do not reconstruct exactly the original image because of the rounding of the floating-point wavelet coefficients to integers caused by the coding. The use of the lifting scheme allows generating truly loss-less non-linear integer-to-integer wavelet transforms. In most of the cases, the filters used in wavelet transforms have floating point coefficients. Since the input images have integer entries, the filtered output no longer consists of integers and losses will result from rounding. For lossless coding it is necessary to make a reversible mapping between an integer image input and an integer wavelet representation. There are two ways to warp an image. The first, called forward mapping, scans through the source image pixel by pixel, and copies them to the appropriate place in the destination image. The second, reverse mapping, goes through the destination image pixel by pixel, and samples the correct pixel from the source image. The most important feature of inverse mapping is that every pixel in the destination image gets set to something appropriate. In the forward mapping case, some pixels in the destination might not get painted, and would have to be interpolated. We calculate the image deformation as a reverse mapping.

\section{Transcoding}

One of the fundamental challenges in deploying multimedia systems, such as telemedicine, education, space endeavors, marketing, crisis management, transportation, and military, is to deliver smooth and uninterruptible flow of audio-visual information, anytime and anywhere. A multimedia system may consist of various devices (PCs, laptops, PDAs, smart phones, etc.) interconnected via heterogeneous wire line and wireless networks. In such systems, multimedia content originally authored and compressed with a certain format may need bit rate adjustment and format conversion in order to allow access by receiving devices with diverse capabilities (display, memory, processing, decoder). Thus, a transcoding mechanism is required to make the content adaptive to the capabilities of diverse networks and client devices. A video transcoder can perform several additional functions. For example, if the bandwidth required for a particular video is fluctuating due to congestion or other causes, a transcoder can provide fine and dynamic adjustments in the bit rate of the video bit stream in the compressed domain without imposing additional functional requirements in the decoder. In addition, a video transcoder can change the coding parameters of the compressed video, adjust spatial and temporal resolution, and modify the video content and/or the coding standard used. Transcoding performs one or more operations, such as bit rate and format conversions, to transform one compressed video stream to another. Transcoding can enable multimedia devices of diverse capabilities and formats to exchange video content on heterogeneous network platforms such as the Internet. One scenario is delivering a high-quality multimedia source (such as a DVD or HDTV) to various receivers (such DATA as PDAs, Pocket PCs, and fast desktop PCs) on wireless and wire line networks. Here, a transcoder (placed at the transmitter, receiver or somewhere in the network) can generate appropriate bit stream threads directly from the original bit stream without having to decode and re-encode. To suit available network bandwidth, a video transcoder can perform dynamic adjustments in the bit-rate of the video bit stream without additional functional requirements in the decoder. Another scenario is a video conferencing system on the Internet in which the participants may be using different terminals. Here, a video transcoder can offer dual functionality: provide video format conversion to enable content exchange, and perform 
dynamic bit rate adjustment to facilitate proper scheduling of network resources. Thus, video transcoding is one of the essential components for current and future multimedia systems that aim to provide universal access. Currently, several video compression standards exist for different multimedia applications. Each standard may be used in a range of applications but is optimized for a limited range. H.261, H.263, H.263 designed by ITU (International Telecommunication Unit) are aimed for low-bit-rate video applications such as videophone and videoconferencing. MPEG standards are defined by ISO (International Organization for Standardization). MPEG-2 is aimed for high bit rate high quality applications such as digital TV broadcasting and DVD, and MPEG-4 is aimed at multimedia applications including streaming video applications on mobile devices. As the number of applications increases and various networks such as wire line and wireless integrate with each other, inter-compatibility between different systems and different platforms are becoming highly desirable. Transcoding is needed both within and across different standards to allow the interoperation of multimedia streams. The first and most important challenge in the context of a video conferencing is to provide transcoding on the fly with real-time speed and without any interruption of video flow.

There are three basic requirements in transcoding,

1) The information in the original bit stream should be exploited as much as possible;

2) The resulting video quality of the new bit stream should be as high as possible, or as close as possible to the bit stream created by coding the original source video at the reduced rate;

3) In real-time applications, the transcoding delay and memory requirement should be minimized to meet real-time constraints.

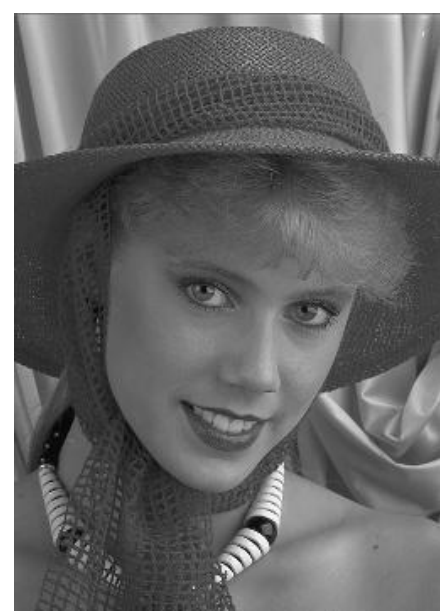

Figure 1. Original Image

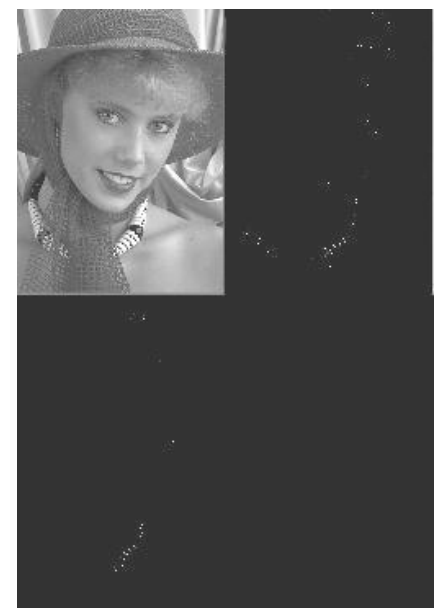

Figure 4. Preprocessed Wavelet Figure
III.

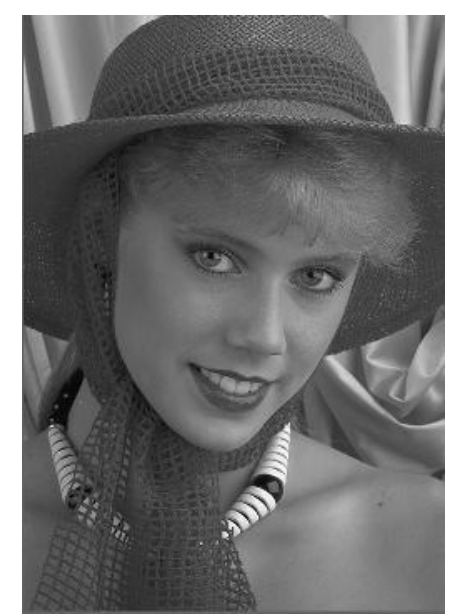

Figure 2. Mosaic Image

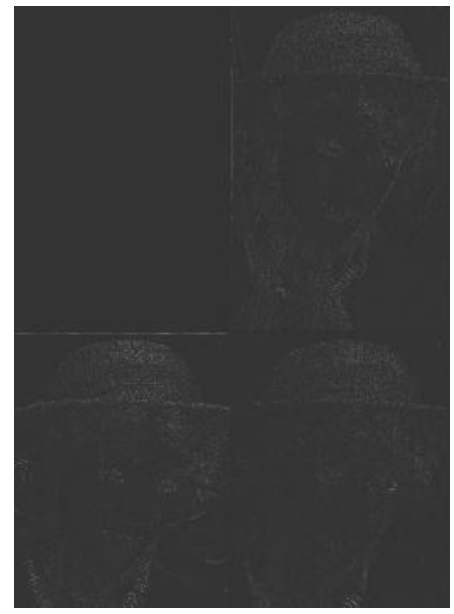

5. Difference between Preprocessed and $5 / 3$ Wavelet

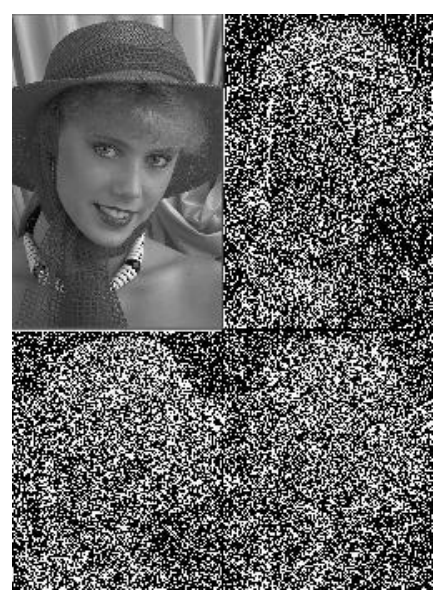

Figure 3. 5/3 Wavelet Transformed Image

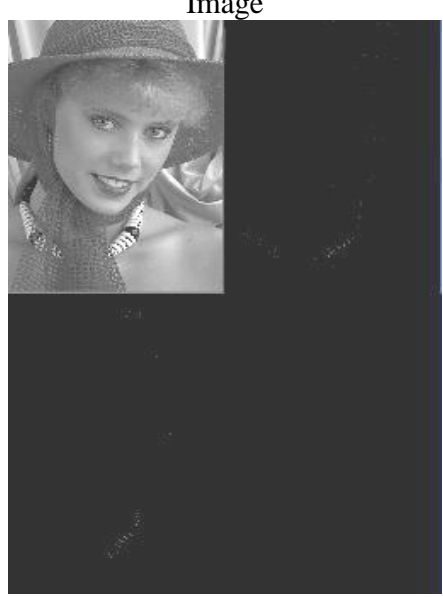

Figure 6. Significant Map 


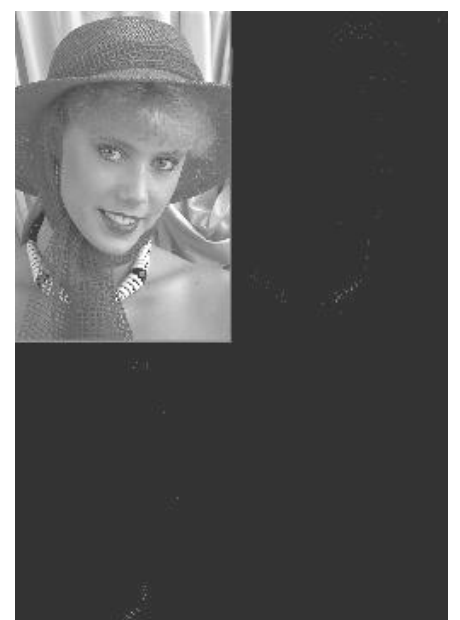

Figure 7. Refinement Map

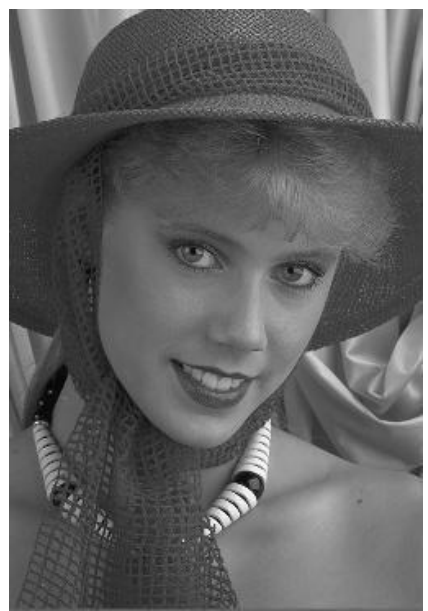

Figure 8. Restored Original Image

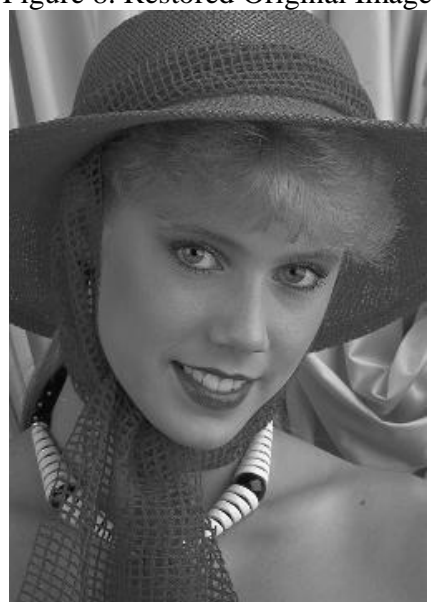

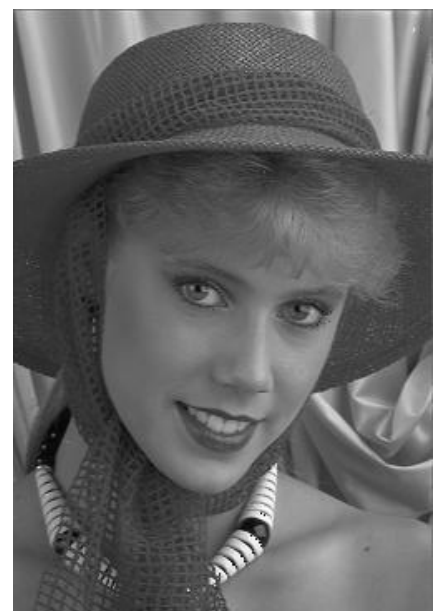

Figure 9. Restored Refined Image

Figure 10. Restored Refined Image Video Output

\section{RESULTS}

1.) PSNR RESULTS OBTAINED WITH TIME SAMPLES

\begin{tabular}{|c|c|}
\hline Time & PSNR \\
\hline 185.456 & 32.6138 \\
\hline 220.127 & 34.8128 \\
\hline 227.397 & 35.1929 \\
\hline 227.467 & 34.188 \\
\hline 229.781 & 34.4217 \\
\hline Time - PSNR Table
\end{tabular}

2.) TIME PSNR GRAPH

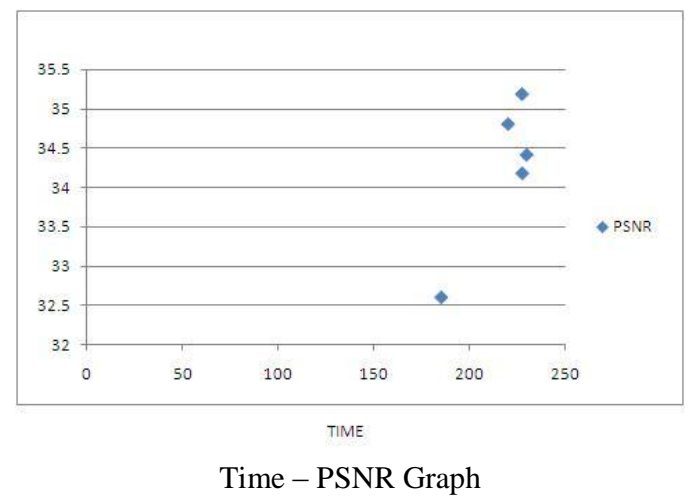

\section{CONCLUSIONS}

Due to the limitations of devices in the networks, Wavelet based compression is the most adopted technique that is used which minimizes the energy required for wireless communication and handles the issues related to the quality of the image when compressed. The $5 / 3$ wavelet transform is adapted as the method as it suits for mosaic images. Mosaicking is 
used when the user intends to include secret information in images and is send to the destination. In order to improve the quality of the image, one or more filtered versions of the image is added to the original image. This technique is known as preprocessing. The problem that is associated when decompressing the images is that, due to the property of floating point nature of wavelet coefficients, the coding effect would round the values to integers. To handle the situation, Refinement Mapping is used. Since the image compressed has to be displayed in a wide variety of devices as chosen by the user, Transcoding is used since it is a bridge between many applications. By Transcoding, the image can be displayed in a wide variety of devices making it more user friendly. Requantization, spatial and temporal downscaling are the most popular Transcoding algorithms. The purpose of the Transcoder is to convert Mpeg-2 standard to Mpeg-4 for supporting mobile devices. It also supports color depth reduction, Text summarization, multi-model, for e.g., text-to-speech, audio driven animation model.

\section{ACKNOWLEDGEMENT}

The authors would like to thank the members of the Dept. of CITE, M S University, Tirunelveli for various algorithms used in this research, and all the people who helped in preparing and carrying out the experiments and data collection.

\section{REFERENCES}

[1]. S.A.Hussain , M.I. Razzak, A. A. Minhas, M. Sher, Energy Efficient Image Compression in wireless Sensor Networks International Journal of Recent Trends in Engineering, Vol 2, No. 1, November 2009

[2]. A.E. Gamal, Collaborative Visual Sensor Networks, 2004, http://mediax.stanford.edu/projects/cvsn.html.

[3]. M. J. Nadenau, J. Reichel, and M. Kunt, "Wavelet Based Color Image Compression: Exploiting the Contrast Sensitivity Function,” IEEE Transactions Image Processing, Vol. 12, no.1, Pp. 58-70, 2003.

[4]. L. Ferrigno, S. Marano, V. Paciello, and A. Pietrosanto, "Balancing computational and transmission power consumption in wireless image sensor networks", IEEE29 International Conference on Virtual Environments, Human- Computer Interfaces, and Measures Systems (VECIMS), Giardini Naxos, Italy, July 2005.

[5]. Min Wu and Chang Wen Chen, "Multiple bitstream image transmission over wireless sensor networks", Proceedings of IEEE Sensors, volume 2, pages 727-731, October 2003.

[6]. Huaming Wu and Alhussein A. Abouzeid, "Energy efficient distributed JPEG2000 image compression in multi hop wireless networks", 4th Workshop on Applications and Services in Wireless Networks (ASWN 2004), pages 152-160, August 2004.

[7]. H.M. Wu, A.A. Abused, Energy, "efficient distributed image compression in resource constrained multichip wireless networks", Computer Communication (Elsevier) 28 (14) (2005) 1658-1668.

[8]. R. Wagner, R. Nowak, and R. Baraniuk."Distributed image compression for sensor networks using correspondence analysis and super-resolution", Proceedings of IEEE International Conference on Image Processing (ICIP), volume 1, pages 597-600, September 2003.

[9]. N. Boulgouris and M. Strintzis. "A family of wavelet-based stereo image coders". IEEE Transactions on Circuits and Systems for Video Technology, 12(10):898-203, October 2002.

[10]. A. Wang. A. Chandrakasan, "Energy efficient system partitioning for distributed wireless sensor networks", Proceedings of the International Conference on Acoustics, Speech, and Signal processing (ICASSP-2001), Salt Lake City, Utah, 2001.

[11]. S.S. Pradhan, J. Kusuma, K. Ramchandran, "Distributed compression in a dense microsensor network", IEEE Signal Processing Magazine 19 (2) (2002) 51-60.

[12]. D. Devaguptapu, B. Krishnamachari, "Applications of localized image processing techniques in wireless sensor networks", SPIE's $17^{\text {th }}$ Annual International Symposium on Aerospace/Defense Sensing, Simulation, and Controls (Aerosense'03), Orlando, Florida, 2003.

[13]. D. Ganesan, B. Greenstein, D. Perelyubskiy, D. Estrin, J.Heidemann, “An evaluation of multiresolution storage for sensor networks", Proceedings of the First International Conference on Embedded Networked Sensor Systems, ACM Press, 2003, pp. 89-102.

[14]. S.D. Servetto, "Sensing lena-massively distributed compression of sensor images", Proceedings of the IEEE International Conference on Image Processing (ICIP) (special session on 'Distributed Source Coding'), Barcelona, Spain, 2003.

[15]. S. Bandyopadhyay, E.J. Coyle, “An energyefficient hierarchical clustering algorithm for wireless sensor networks”, INFOCOM, 2003.

[16]. Vincent Lecuire, Cristian Duran-Faundez, and Nicolas Krommenacker, "Energy-Efficient Transmission of Wavelet-Based Images in Wireless Sensor Networks", Eurasip journal on Image and Video Processing, 11 pages, 2007.

[17]. H. Wu, A.A. Abouzeid, "Energy efficient distributed JPEG2000 image compression in multihop wireless networks" ASWN 2004. pp 152- 160.

[18]. C. Ben Amar, and O. Jemai, "Wavelet Networks Approach for Image Compression," ICGST, 2003.

[19]. V. Singh, N. Rajpal, and K. S. Murthy, "Neuro-Wavelet Based Approach for Image Compression," Computer Graphics, Imaging and Visualization, CGIV apos'07, Pp. 280-286, 2007.

[20]. Jung-Hua Wang, and Ker-Jiang Gou, "Image compression using wavelet transform and self-development neural network,” IEEE International Conference on Systems, Man, and Cybernetics, Vol. 4, Pp. 4104-4108, 1998. 
T. Arumuga Maria Devi received B.E. Degree in Electronic and Communication Engineering from Manonmaniam Sundaranar University, Tirunelveli India in 2003, M.Tech degree in Computer and Information Technology from Manonmaniam Sundaranar University, Tirunelveli, India in 2005. Currently, she completed Ph.D in Computer and Information Technology and is also the Assistant Professor of Centre for Information Technology and Engineering of Manonmaniam Sundaranar University. Her research interests include Signal and Image Processing, Multimedia and Remote Communication.

Sherin K K, Assistant Professor, received M.Tech Degree in Computer and Information Technology from Manonmaniam Sundaranar University, Tirunelveli, India in 2011,

M Sc degree in Software Engineering from N I College of Engg, Affliated to Anna University Chennai, India in 2006, M.Phil degree in Information Technology from Manonmaniam Sundaranar University, Tirunelveli, India in 2012. His research interest includes Digital Image Processing, Remote Communication and Artificial Intelligence. 